\title{
Comparative Analysis of Original Wave \& Filtered Wave of EEG signal Used in the Prognostic of Bruxism medical Sleepsyndrome
}

\author{
Md Belal Bin Heyat \\ Department of ECE \\ Glocal University \\ Saharanpur, India
}

\author{
Faijan Akhtar \\ Department of CSE \\ Jamia Hamdard \\ New Delhi, India
}

\author{
Shadab Azad \\ Department of Unani \\ Ajmal Khan Tibbia College \\ Muzaffarnagar, India
}

\begin{abstract}
The bruxism is a medical sleep syndrome; it is the remedial span for crushing the tines and gritting the jowl. Human rarely chore their tines and jowl, slightly than crushing their teeth lacking it producing any signals. The symptoms of bruxism are arduousness in the jowl joint, breakable teeth, headache, earache and difficulty in open in mouth etc. The causes of bruxism are snooze sickness, pressure and nervousness. The REM is a rapid eye movement; it's a stages of sleep. The EEG signal are used in the measurement of neuron, the alpha, beta, gamma, theta and delta wave are used in the prognostic of bruxism syndrome. It's used in MATLAB coding by the six steps in prognostic in bruxism.
\end{abstract}

Keywords: Bruxism;EEG signal; REM

\section{INTRODUCTION}

Bruxism is the remedial span for crushing the tines and gritting the jowl. Persons occasionally chore their tines lacking it producing any indications. However, even, tenacious tines crushing can origin jowl pain and distress and attire dejected your tines. Bruxism also touches people when they are wakeful, even though this is additional probable to be compressing the tines and jowl, slightly than crushing their tines. Most publics prepare it subconsciously although engaged or when they are in traumatic circumstances. The eighty percent bruxism is found in sleep time i.e. called as bruxism sleep disorder.

\section{Symptoms of Bruxism}

- Arduousness in the jowl joint

- Cracked teeth

- Difficulty in opening your mouth

- Earache

- Headaches

- Makeover muscle pain
- Snooze disturbance

- Tautness in the shoulders

\section{Causes of Bruxism}

- Sleep sickness

- Pressure

- Nervousness

\section{REM}

REM is a Rapid Eye Movement, It's a sleep stage. It is a final stage of Sleep stage, the total number of sleep time ninety minute to one hundred twenty minute during the night. In this stage up to twenty percent to twenty five percent in full, sleep time in adult's humans. Rapid Eye Movement is a single stage of mammalian snooze considered by casual movement of the senses, low influence tone during the figure, and the tendency of sleeper toward vision intensely. This stage is also recognized as absurdsnooze and occasionally desynchronized snooze since of bodilyresemblances to awakening states, countingquick, stumpy powerdesynchronized mindsprays.Rapid Eye Movement snooze is interrupted and directly heralded by Ponto Geniculo Occipital sprays, eruptions of electrical movement inventing in the mind twig. These sprays occur in knots nearby every six seconds for one minute to two minutes thru the conversion from profound to absurd snooze.

\section{EEG}

EEG is known as electroencephalogram signal,Electroencephalogram paths and archives mind wave designs. Minor metal records with electrodes are 
located on the scalp then refer indications to a processer to record the outcomes. Common electrical movement in the mind makes an identifiable design. Complete Electroencephalogram; medics can aspect for irregular designs that indicate commandeering and other difficulties. Some EEG waves are found in the brain mapping-

\begin{tabular}{|c|c|c|c|}
\hline $\begin{array}{c}\text { S. } \\
\text { No. }\end{array}$ & $\begin{array}{c}\text { Name of } \\
\text { EEG Wave }\end{array}$ & $\begin{array}{c}\text { Amplitude } \\
(\mu \mathrm{V})\end{array}$ & $\begin{array}{c}\text { Frequency } \\
(\mathrm{Hz})\end{array}$ \\
\hline $\mathbf{1}$ & Alpha & 2 to 10 & 8 to 13 \\
\hline $\mathbf{2}$ & Beta & 1 to 5 & 13 to 22 \\
\hline $\mathbf{3}$ & Gamma & 10 to 20 & 22 to 30 \\
\hline $\mathbf{4}$ & Delta & 20 to 400 & 0.5 to 4 \\
\hline $\mathbf{5}$ & Theta & 100 to 500 & 4 to 8 \\
\hline
\end{tabular}

Table 1: EEG waves with amplitude \& frequency [6]

\section{RESULT}

The prognostic of bruxism medical sleep disorder is done by MATLAB coding; the prognostic of bruxism medical sleep syndrome is done by different step-

$>$ The first one is data of the patient and normal human is downloaded $\mathrm{n}$.mat file and info file in one-minute time.

$>$ The second one is downloaded data is loaded by MATLAB coding. The output of the loaded data is found to all channels.

$>$ The third one is cut the EMG1-EMG2 channel to all channels of electroencephalogram signals.

$>$ The fourth one is EMG1-EMG2 channel is passed by low pass filter.

$>$ The fifth one is hamming window is apply.

$>$ The sixth one is compare by original wave and filtered wave shown in figure.

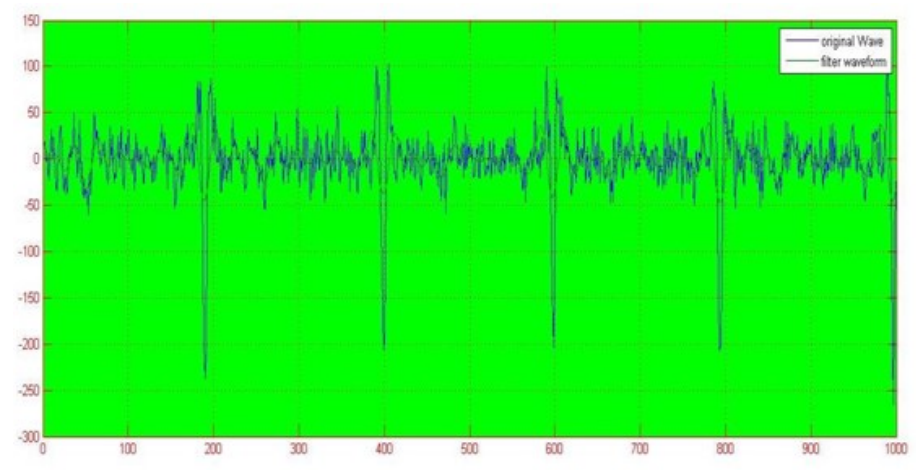

Fig 1: Comparative EEG signal of EMG1EMG2 channel in Bruxism patient

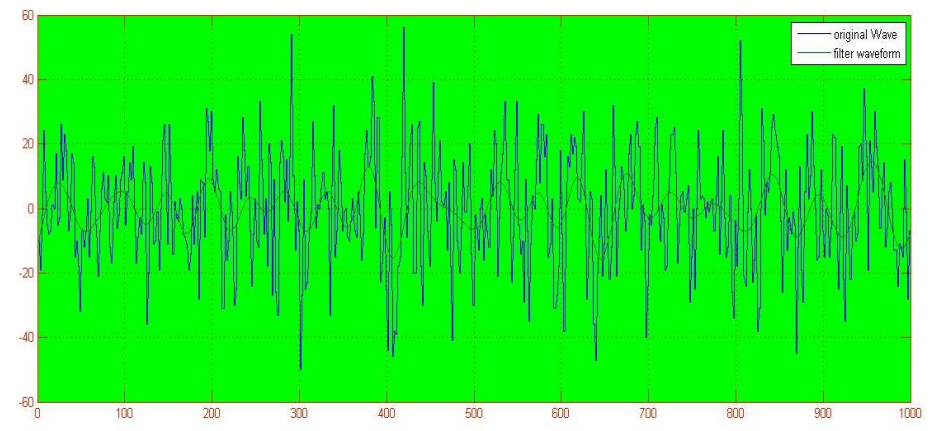

Fig 2: Comparative EEG signal of EMG1-EMG2 channel in Normal human

The above figure 1 and 2 is clear that the prognostic of bruxism sleep syndrome.

\section{CONCLUSION}

The Prognostic of sleep bruxism medical syndrome is clear in the fig 1 and 2. The MATLAB coding technique is best in the prognostic of bruxism sleep medical syndrome.On future work, the comparative signal is converted in the digital form.

Abbreviations- EEG: Electroencephalogram; EMG: Electromyogram; REM: Rapid Eye Movement.

Competing interests- Md Belal Bin Heyat is a researcher and author asserts that they have no competing interests.

\section{REFERENCES}

[1] www.nhs.uk

[2]blog.withings.com

[3]kidshealth.org

[4]en.wikipedia.org 
[5] Er. ShipraSrivastava, Mohd Maroof Siddiqui, SaifurRahman, Prof (Dr.) Syed HasanSaeed, Md Belal Bin Heyat, "Carbon Nano tubes \& Its Application In Medical Field \& Communication", International Journal of Advanced Research in Computer and Communication Engineering 2016 May;5(5): pp.170-173.

[6] Md Belal Bin Heyat, Shaguftah, Y.M.Hasan, M.M.Siddiqui, "EEG Signals and Wireless transfer of EEG Signals", International Journal of Advanced Research in Computer and Communication Engineering Volume 2015 December;4(12): pp. 502-504.

[7] Md Belal Bin Heyat, Faijan Akhtar, Mohd Sikandar Hayat Siddiqui, Shafan Azad, "An Overview of Dalk Therapy and treatment of Insomnia by Dalk Therapy", National Seminar on Research Methodology in Ilaj-BitTadbeer, on State Takmeel-ut-Tib-College \& Hospital, Lucknow, 10 October 2015.

[8] Y.M.Hasan, Md Belal Bin Heyat, M.M.Siddiqui, Shafan Azad, and Faijan Akhtar, "An Overview of Sleep and Stages of Sleep", International Journal of Advanced Research in Computer and Communication Engineering, 2015 December;4(12): pp. 505-507.

[9] Md Belal Bin Heyat, Mohd Maroof Siddiqui, "Recording of EEG, ECG, EMG Signal", International Journal of Advanced Research in Computer Science and Software Engineering, 2015 October;5(10): pp. 813-815.

[10] Md Belal Bin Heyat, Mohd Maroof Siddiqui, Faijan Akhtar, "An Overview of NFLE", International Journal of Innovative Research in Electrical, Electronics, Instrumentation \& Control Engg, 2016 March;4(3): pp. 209-211.

[11] Touseef Rahman, Omer Farook, Md Belal Bin Heyat, Mohd Maroof Siddiqui, "An Overview of Narcolepsy", International Advanced Research Journal in Science, Engineering and Technology, 2016 March;3(3):pp. 85-87.

[12] Md Belal Bin Heyat, Faijan Akhtar, Shaguftah, Naseem Ahmad, "An Overview of Renewable Energy",International Journal of Technical Research \& Science, 2016 September;1(6): pp. 119-121.

[13] Md Belal Bin Heyat, et al. "Microcontroller Using Industrial Tank", Onyx Journal of Multi- Disciplines, Onyx InterScience, 2016 October;1(1):pp. 5-8.

[14] Md Belal Bin Heyat, Shaguftah, Faijan Akhtar, Mohd Ammar Bin Hayat, Shajan Azad, "Power Spectral Density are used in the Investigation of insomnia neurological disorder", XL PRE-CONGRESS Symposium, on INDIAN ACADEMY OF SOCIAL SCIENCES [ISSA] in King
George Medical University \& State Takmeelut-tib College and Hospital Lucknow.

[15] Shahabaz Ahmad Khan, Ahmad Zakariya, ErSaima Beg, Md Belal Bin Heyat and Mohd Maroof Siddiqui, "Industrial Tank Temperature, Pressure and Humidity Controller Using Microcontroller", National Conference on Emerging Trends in Non Conventional Energy Resources on Integral University, Lucknow, 22 October 2016.

[16] Md Belal Bin Heyat, et al. "A Review on use of Sunlight in Human Life", International Journal of Trend in Scientific Research and Development, 2016 Nov-Dec;1(2): pp. 22-24.

[17] Md Belal Bin Heyat, "Insomnia: Medical Sleep Disorder \& Diagnosis", Anchor Academic Publishing, Hamburg, Germany 2016.

[18] Siddiqui, Mohd Maroof, GeetikaSrivastava, and Syed HasanSaeed. "Detection of rapid eye movement behaviour disorder using short time frequency analysis of PSD approach applied on EEG signal (ROC-LOC)." Biomedical Research 26.3 (2015): 587-593.

[19]Sumbul Mehdi, Md Belal Bin Heyat, Faijan Akhtar, Mohd Ammar Bin Heyat and Tanya Gupta, "Cure of Epilepsy by Different System of Medicine", International Journal of Technical Research \& Science, 2016 November;1(8): pp. 244-247. 